\title{
How to Intervene in the Caries Process in Children: A Joint ORCA and EFCD Expert Delphi Consensus Statement
}

\author{
Christian H. Splieth $^{\mathrm{a}} \quad$ Avijit Banerjee $^{\mathrm{b}}$ Peter Bottenberg ${ }^{\mathrm{c}}$ Lorenzo Breschi $^{\mathrm{d}}$ \\ Guglielmo Campus ${ }^{\mathrm{e}}{ }^{\mathrm{f}}$ Kim Rud Ekstrand ${ }^{g}$ Rodrigo A. Giacaman ${ }^{\mathrm{h}}$ Rainer Haak ${ }^{\mathrm{i}}$ \\ Matthias Hannig ${ }^{j}$ Reinhard Hickel ${ }^{k}$ Hrvoje Juric' ${ }^{\prime}$ Adrian Lussi ${ }^{m, n}$ \\ Vita Machiulskiene $^{\circ} \quad$ David J. Manton $^{p, q}$ Anahita Jablonski-Momeni ${ }^{r}$ \\ Niek J.M. Opdam ${ }^{\mathrm{s}}$ Sebastian Paris ${ }^{\mathrm{t}}$ Ruth M. Santamaría ${ }^{\mathrm{a}}$ Falk Schwendicke ${ }^{\mathrm{u}}$ \\ Herve Tassery ${ }^{\mathrm{v}, \mathrm{w}}$ Andrea Ferreira Zandona ${ }^{\mathrm{x}}$ Domenick T. Zero ${ }^{\mathrm{y}}$ \\ Stefan Zimmer ${ }^{Z}$ Sophie Doméjean ${ }^{\mathrm{A}, \mathrm{B}}$
}

\begin{abstract}
a Preventive and Pediatric Dentistry, Center for Oral Health, Universitätsmedizin Greifswald, Greifswald, Germany; ${ }^{b}$ Conservative \& MI Dentistry, Faculty of Dentistry, Oral \& Craniofacial Sciences, King's College London, London, UK; ' Oral Health Research Group, Vrije Universiteit Brussel, Brussel, Belgium; ${ }^{\mathrm{d} D e p a r t m e n t ~ o f ~ B i o m e d i c a l ~ a n d ~}$ Neuromotor Sciences, DIBINEM, University of Bologna - Alma Mater Studiorum, Bologna, Italy; ${ }^{e}$ Department of Restorative, Preventive and Pediatric Dentistry, Zahnmedizinische Kliniken (ZMK), University of Bern, Bern, Switzerland; ${ }^{f}$ Department of Surgery, Microsurgery and Medicine Sciences, School of Dentistry, University of Sassari, Sassari, Italy; ${ }^{9}$ Department of Odontology, University of Copenhagen, Copenhagen, Denmark; ${ }^{\mathrm{h}}$ Cariology Unit, Department of Oral Rehabilitation, Faculty of Health Sciences, University of Talca, Talca, Chile; 'Department of Cariology, Endodontology and Periodontology, University Leipzig, Leipzig, Germany; ${ }^{j}$ Clinic of Operative Dentistry, Periodontology and Preventive Dentistry, Saarland University, Homburg, Germany; ${ }^{k}$ Department of Conservative Dentistry and Periodontology, University Hospital, LMU Munich, Munich, Germany; 'Department of Pediatric and Preventive Dentistry, School of Dental Medicine, University of Zagreb, Zagreb, Croatia; ${ }^{\mathrm{m} S c h o o l}$ of Dental Medicine, University of Bern, Bern, Switzerland; ${ }^{\mathrm{n}}$ Department of Operative Dentistry and Periodontology, Faculty of Dentistry, University Medical Centre Freiburg, Freiburg, Germany; ${ }^{\circ} \mathrm{Clinic}$ of Dental and Oral Pathology, Faculty of Odontology, Lithuanian University of Health Sciences, Kaunas, Lithuania; ${ }^{P}$ Centrum voor Tandheelkunde en Mondzorgkunde, Universitair Medisch Centrum Groningen, University of Groningen, Groningen, The Netherlands; ${ }^{\mathrm{q}}$ Melbourne Dental School, University of Melbourne, Melbourne, VIC, Australia; ' ${ }^{\mathrm{D}}$ Department of Orthodontics, Dental School, Philipps University Marburg, Marburg, Germany; ${ }^{5}$ Department of Dentistry, Radboud Institute for Health Sciences, Radboud University Medical Center, Nijmegen, The Netherlands; ${ }^{\mathrm{t}}$ Department of Operative and Preventive Dentistry, Charité - Universitätsmedizin Berlin, Berlin, Germany; ${ }^{\text {u} D e p a r t m e n t ~ o f ~ O r a l ~ D i a g n o s i s, ~ D i g i t a l ~}$ Health and Health Services Research, Charité - Universitätsmedizin Berlin, Berlin, Germany; vEA 4203 Laboratory, Faculté d'Odontologie, Université de Montpellier, Montpellier, France; ${ }^{w}$ Faculté d'Odontologie Marseille, Preventive and Restorative Department, Aix-Marseille-Université, Marseille, France; ${ }^{\times}$Department of Comprehensive Care, Tufts University School of Dental Medicine, Boston, MA, USA; ${ }^{y}$ Oral Health Research Institute, Indiana University School

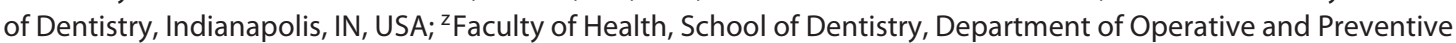
Dentistry, Witten/Herdecke University, Witten, Germany; ${ }^{A}$ Centre de Recherche en Odontologie Clinique EA 4847, UFR d'Odontologie, Département d'Odontologie Conservatrice, Université Clermont Auvergne, Clermont-Ferrand, France; ${ }^{B}$ Service d'Odontologie, CHU Estaing Clermont-Ferrand, Clermont-Ferrand, France
\end{abstract}

C.H. Splieth

Preventive and Pediatric Dentistry

University of Greifswald, Fleischmannstrasse 42

DE-17487 Greifswald (Germany)

splieth@uni-greifswald.de 


\section{Keywords}

Children - Caries treatment - Early childhood caries - Primary teeth $\cdot$ Permanent molars $\cdot$ Fluoride $\cdot$ Sealant $\cdot$ Restoration dren, i.e., early childhood caries (ECC), caries in primary molars, and occlusal caries in the permanent dentition of children and adolescents.

\section{Background}

Caries is currently defined as "a biofilm-mediated, diet-modulated, multifactorial, noncommunicable, dynamic disease resulting in net mineral loss of dental hard tissues. It is determined by biological, behavioral, psychosocial and environmental factors. As a consequence of this process, a caries lesion develops" [Machiulskiene et al., 2020]. This definition allows for a very broad range of interventions in the caries process, from primary prevention to extractions. Caries care, management, and control are actions taken to interfere with mineral loss at all stages of the caries process [Nyvad and Fejerskov, 2015], including nonoperative and operative interventions.

Especially in children, there are multiple options due to the eruption of a second emerging dentition. The decision-making and final treatment approach is influenced not only by the staging of a single caries lesion but rather by multiple factors, particularly in the case of ECC or caries in the primary dentition in general in those who are very young, often presenting with multiple caries lesions, pulpal involvement, and insufficient cooperation.

The overall caries decline is associated with an increasing polarization in caries distribution; while many children are free of caries defects, a minority of highly cariesactive children, from an often low socioeconomic background, remains in many high-income countries [Pitts et al., 2019]. In Germany, for example, the entire caries experience on the defect level $\left(\mathrm{d}_{3-4} \mathrm{mft} / \mathrm{D}_{3-4} \mathrm{MFT}\right)$ is concentrated in only $14 \%$ of 3 -year-olds and $19 \%$ of 12 -yearolds, with a high mean of multiple and often untreated lesions [Team DAJ, 2017]. Consequently, different preventive and treatment interventions may be required for such high-caries-risk groups compared to the majority of the population. In countries with a generally high caries prevalence, the high caries risk group will be larger and in these instances a population-based approach without adjustment for caries risk may be advisable.

With the eruption of the first permanent molar, the occlusal surface becomes an important predilection site for the development of caries lesions, being one of the most frequently restored sites [Carvalho et al., 2016]. The occlusal surface, therefore, requires intensified efforts for preventive, nonoperative/noninvasive, micro- or minimally invasive and restorative interventions according to 
the level of caries severity and the caries activity/risk status of the patient. This paper condenses the available evidence to provide clinical recommendations for caries management in children.

\section{Materials and Methods}

After agreement on the need for this consensus process, participants were selected and invited by the boards of the EFCD, the ORCA, and the DGZ aiming for a well-balanced representation of experts in the fields of cariology, restorative dentistry, pediatric dentistry, and gerodontology.

The basis for the consensus were 6 systematic reviews on various aspects of caries in children, adults, and older persons authored by mixed teams appointed from the consensus group of the ORCA and EFCD/DGZ. The reviews on ECC, caries in primary teeth, and occlusal caries in permanent molars [Santamaria et al., 2020; Schmoeckel et al., 2020; Campus et al., 2020] were coordinated by C.H.S. and the aim was to answer the following PICO (patient/population/problem, intervention, comparison, outcome) questions:

1 How should one intervene in ECC (especially upper anterior teeth)?

(a) What is the effect of noninvasive treatment options on ECC?

(b) What are the success rates of invasive/operative treatment options of ECC (e.g. filling, endodontic treatment, crown, extraction)?

2 How should one intervene in children with caries lesions in primary molars?

3 How should one intervene in children (and adolescents) with occlusal caries lesions in permanent teeth?

Based on these 3 systematic reviews on caries in children and adolescents, as well as other literature, structured consensus statements and clinical recommendations were drafted by the group chairs (C.H.S., S.P., and F.S.) and sent to the delegates prior to the workshop meeting in Berlin, Germany, on June 13, 2019.

For the general and preventive statements, other systematic reviews and guidelines were considered. In the subsequent joint e-Delphi workshop, the final wording of the statements was developed trying to include aspects from the fields of cariology and restorative, pediatric, and preventive as well as community dentistry. While the majority of the consensus committee attended the workshop in Berlin, some members (P.B., G.C., K.R.E., H.J., H.T., A.F.Z., D.T.Z., and D.J.M.) were not able to attend the conference, but they were equally involved in the subsequent voting process as well as in the writing of this paper.

At the Berlin meeting, each statement was extensively discussed and modified until a consensus was reached. The strength of each recommendation was evaluated by the group and categorized as "strong," "moderate," or "weak" based on the scientific evidence supporting the statement. Recommendations supported by unequivocal evidence (e.g., many randomized controlled trials) were evaluated as "strong." Recommendations based on moderate evidence (e.g., high-quality clinical studies, such as randomized controlled trials with similar results) were evaluated as "moderate." Finally, recommendations based on expert opinion only and those that are based on weak evidence (e.g., no clinical studies or only low-quality studies or studies with contradicting results) were ranked as "weak."

Based on the discussions at the meeting, this paper was drafted by the session chair for the children subgroup (C.H.S.), revised by the steering group of the consensus process (S.P., C.H.S., and F.S.), and sent to the overall group, who commented on it extensively, in 2 rounds. Afterwards, a confidential e-Delphi survey was finally undertaken and each single recommendation, as well as the corresponding strength of the recommendation, was ranked by each participant. The text itself (excluding the recommendations) was not submitted to any further consensus process as it was felt that the core of the consensus was the recommendations.

The voting on the statement was graded from 1 (completely disagree) to 10 (completely agree). At least $70 \%$ of the vote over 7 was considered as acceptance of the statement by the group and the results were reported as agreement (i.e., 10 to 8), neutral (i.e., 7 to 4 ), or disagreement ( 3 to 1 ). In addition, the median of all of the votes was calculated. An additional field for free-text comments was also available to allow the reasoning for a certain decision or proposals for future modifications.

\section{Results}

\section{Caries Patterns in Children}

The process of growth and development in children, their immature intellectual, emotional and psychological status, the dependence on caregivers, and a second emerging dentition pose several challenges beyond the staging of caries lesions that play a crucial role in the decision making of how to intervene in the caries process in children, especially for the primary dentition.

In addition, the polarization of the caries distribution in countries that have achieved a major caries decline leads to a majority of children with few or even no detectable caries lesions, while a minority group still exhibits extensive tooth damage due to caries. This is especially obvious in ECC with its early onset of caries in young children with an often fast progression, which can result finally in complete destruction of the primary dentition. Due to the frequent consumption of carbohydrates, especially sugars, and inadequate to absent oral hygiene in young children, ECC demonstrates an atypical pattern of caries attack, particularly on smooth surfaces of upper anterior teeth [Machiulskiene et al., 2020].

Due to the specific morphology of primary teeth with thinner enamel and dentine layers as well as proportionally larger pulp chambers, caries compromises the pulp earlier, causing a significant pain problem in young, usually precooperative children, often requiring sedation or even general anesthesia (GA) for adequate treatment. Thus, ECC is a highly prevalent, but often neglect- 
ed, global burden. Nationally representative caries surveys for 1-, 2-, or 3-year-olds are often missing, but where they have been performed a higher prevalence and severity have been found in these young children compared to the permanent dentition, which is almost completely untreated. The prevalence ranges from $10 \%$ to more than half of the children [Ganesh et al., 2019; Tinanoff et al., 2019], and it is mostly linked to a low socioeconomic status and its severe forms can be considered child neglect.

\section{Clinical Recommendations}

Due to the transitional function of the primary dentition, the often reduced compliance of young children, the involvement of multiple teeth or lesions, and the growth and development of the permanent dentition, the treatment of ECC or caries in children and adolescents in general is defined not only by lesion depth, activity, and optimal restoration but also by relevant overall factors such as ensuring a functional dentition, surrounding structures and facilities, and social, psychological, and financial parameters. The primary goal is not the restoration of single lesions but rather control of the overall caries activity and stabilization of the general oral function and wellbeing. This can lead, e.g., to extractions of restorable teeth if appropriate due to overarching reasons (strength of recommendation: weak; consensus: agreement $88 \%$, neutral $12 \%$, disagreement $0 \%$; median: 9).

Due to the caries decline and the subsequent polarized distribution in children in many countries, future measures for controlling caries have to concentrate on the children that are not sufficiently affected by this caries decline. These are the children exhibiting mostly insufficient oral home care, persistent high caries activity, multiple carious defects, and an often low socioeconomic status. For the control of caries in the remaining children, which are the clear majority, the established programs that have led to a remarkable caries reduction have to be maintained (strength of recommendation: weak; consensus: agreement $96 \%$, neutral $4 \%$, disagreement $0 \%$; median: 10).

\section{Early Childhood Caries}

\section{Prevention of ECC}

The etiology of ECC has been clear for many years and presents globally with identical patterns [Adair et al., 2004], i.e., a high intake of dietary sugars often via the nursing bottle and insufficient or no oral hygiene practices leading to a relatively atypical, fast progressing caries attack starting with the smooth surfaces of the upper anterior teeth in young children [Wyne, 1999]. This definition reveals potentially successful approaches for a prevention or control of ECC. A recent systematic review on prenatal preventive programs found that education of parents on the nature of ECC, recommendations for sugars control, and oral hygiene measures in children significantly reduced the incidence of ECC [Xiao et al., 2019]. This is in line with standard cariespreventive approaches, but the evidence for these programs regarding ECC is limited due a considerable risk of bias caused by the representativity of the selected groups for this very early intervention, ethical concerns with a nonintervention control group, and examination of mostly multiple measures in combination. In addition, it is a problem that a considerable number of parents are not aware of these standard preventive procedures, especially brushing from the eruption of the first tooth onwards.

As fluorides have proven especially successful in the prevention of caries, they should be used from early childhood [Walsh et al., 2019]. To limit the risk of fluorosis in young children and due to the primarily topical action of fluorides, national guidelines favor toothbrushing with an age-adjusted concentration and amount of fluoridated toothpaste. Additional preventive fluoride varnish applications are also effective in achieving a positive cost-benefit ratio for groups or populations with a high caries prevalence [Schwendicke et al., 2018; Toumba et al., 2019].

\section{Preventive Recommendations}

Before birth or/and until the eruption of the first tooth, parents should be informed about the etiology of ECC and the importance of a restricted frequency of sugar intake. They should be trained in adequate oral hygiene and they should perform and support this oral hygiene daily until the child is able to perform it adequately on his or her own (strength of recommendation: moderate; consensus: agreement $100 \%$, neutral $0 \%$, disagreement $0 \%$; median: 10).

Children's teeth should be brushed from the eruption of the first tooth daily with fluoridated toothpaste. Brushing should be performed by parents or caregivers. In the absence of other relevant fluoride sources, especially fluoridated drinking water, the toothpaste should contain $\geq 1,000 \mathrm{ppm}$ fluoride (strength of recommendation: strong; consensus: agreement $92 \%$, neutral $8 \%$, disagreement $0 \%$; median: 10 ). 
Preventive high-concentration fluoride varnish applications should be administered in children, groups, or populations with high caries risks/prevalences in young children 2-4 times a year (strength of recommendation: strong; consensus: agreement $96 \%$, neutral $0 \%$, disagreement 4\%; median: 9).

\section{Treatment for ECC}

As the high frequency of sugar intake is one of the main etiological problems in ECC [Adair et al., 2004], it seems advisable to control the consumption of sugars. Clinically, the first signs of ECC are noncavitated caries lesions or very superficial cavitated dentine lesions on the smooth surfaces of the upper anterior teeth with reversible pulpal inflammation and hypersensitivity restricted to a stimulus without spontaneous or persisting pain.

There is a moderate level of evidence that fluoride varnish accompanied by improving home toothbrushing with fluoridated toothpaste is effective in arresting or remineralizing especially noncavitated lesions, while 5 systematic reviews showed a high level of evidence for arresting even cavitated dentine lesions in ECC with silver diammine fluoride [Schmoeckel et al., 2020].

\section{Noninvasive Treatment Recommendations}

In children with ECC, high frequencies of sugar intakes via food or drinks should be reduced (strength of recommendation: weak; consensus: agreement $100 \%$, neutral $0 \%$, disagreement $0 \%$; median: 10 ).

Noncavitated ECC lesions should be primarily treated with nonoperative measures such as improving home toothbrushing with fluoridated toothpaste and additional fluoride varnish applications (strength of recommendation: moderate; consensus: agreement $92 \%$, neutral $8 \%$, disagreement $0 \%$; median: 10 ).

Dentine lesions in ECC without pulpal involvement can be treated successfully with silver (diammine) fluoride $(\geq 30 \%)$. A side-effect is black staining of the carious dentine (strength of recommendation: high; consensus: agreement $88 \%$, neutral $12 \%$, disagreement $0 \%$; median: 9).

Regarding restorative or invasive treatment approaches in cavitated lesions of anterior upper primary teeth comparative studies with nonoperative treatment, with various restorative techniques or versus extractions, are lacking. This makes the formulation of valid clinical recommendations difficult or impossible and calls for highquality comparative studies or at least systematic followups of treatment cohorts. For existing cohort studies on ECC treatment provided under GA, results vary from low to unacceptable, with annual failure rates of restorations from 5 to $25 \%$ at the tooth level, meaning highly probable complications in children with multiple teeth involved. There is a low level of evidence for higher failure rates of glass ionomer cement (GIC) and resin composite fillings even if they are placed under optimal technical conditions (GA) than for resin composite strip crowns and other preformed crowns [Walia et al., 2014].

\section{Invasive Treatment Recommendations}

Composite strip crowns or other preformed crowns should be preferred over direct fillings (GIC, composite, and others) in anterior upper primary teeth of children with severe ECC, also when placed under GA (strength of recommendation: weak; consensus: agreement $61 \%$, neutral 27\%, disagreement 12\%; median: 7).

Due to the often persisting high caries activity in children, the multiple lesions, and limited cooperation, caries control in these children should consist of robust measures with high success rates including extractions as low compliance can be expected. This applies especially for treatment performed under GA (strength of recommendation: weak; consensus: agreement $77 \%$, neutral $12 \%$, disagreement $8 \% *$; median: 8 ; ${ }^{*} \neq 100 \%$ due to rounding).

Severe ECC has a marked negative influence on the quality of life in preschool children [Collado et al., 2017]. Due to the young age and resulting poor cooperation, highly invasive dental treatment such as pulp therapy, preformed metal crowns, or even extraction can often only be provided under GA at considerable costs [Casamassimo et al., 2009; Thomson, 2016]. Therefore, decision making on how to intervene in children with ECC is more complex than the diagnosis at tooth level. Due to the often complete dentition being affected by ECC at various levels of lesion severity, a patient-centered, comprehensive management protocol for controlling caries activity and ensuring oral rehabilitation has to be found.

\section{Invasive Treatment Recommendation}

Due to the high failure rate of restorations in the upper anterior teeth of children with severe ECC and the lacking function as space maintainer for the permanent dentition, the extraction of upper anteriors can be a justified therapy. A replacement, especially if a removable space maintainer is incorporated for missing primary molars, can be considered, especially for the development of proper orofacial function (strength of recommendation: weak; consensus: agreement $71 \%$, neutral $20 \%$, disagreement $8 \% *$; median: $8 ;^{*} \neq 100 \%$ due to rounding). 


\section{Caries in Primary Teeth}

Apart from ECC, the primary dentition in general seems to be more prone to caries development, as the caries experience in first 5-7 years of the primary dentition generally exceeds the caries increment for the following 6 years of the developing permanent dentition [Santamaria et al., 2019; Splieth et al., 2019].

For primary prevention in the primary dentition, all prophylactic measures described under the subheading "Prevention of ECC" such as biofilm control via toothbrushing, fluoridated toothpaste, and control of the sugar intake can be clinically used. In the case of a higher caries risk or activity, further interventions such as fluoride varnish applications can be performed.

Occlusal caries defects in the primary dentition, a limited number of caries lesions or lesions in the late mixed dentition can be treated with defect-orientated adhesive resin composite, compomer-based or resin-modified GIC fillings, especially in countries or groups with established health care systems that ensure adequate caries prevention and, therefore, a low caries activity [Qvist et al., 2004].

Unfortunately, a high caries prevalence and unsatisfactory care indices in the primary dentition can be found in many countries [Pitts et al., 2019]. Here, the primary molars are of special concern, as they exfoliate much later than incisors and have a space-maintaining function for the permanent dentition. Due to the thinner enamel and more voluminous pulp size, the scientific literature concentrates on the treatment of dentine lesions with nonoperative versus restorative techniques as well as selective ("incomplete or partial"), nonselective ("complete"), and no carious tissue removal [Santamaria et al., 2020]. A recent summary of 2 systematic reviews and 5 randomized clinical trials assessing the effectiveness of interventions, and 10 systematic reviews and 1 RCT assessing the success rates of different dental materials revealed that for treatments of asymptomatic moderate to deep lesions involving no carious tissue removal, the Hall technique (HT) showed lower restoration failure for multisurface cavities as compared to traditional nonselective carious tissue removal and restoration.

The high success rates of the HT are also achieved by the more invasive classical preparations for preformed metal crowns [Innes et al., 2015], also often requiring an accompanying pulpotomy. Nonrestorative caries control (NRCC) showed results comparable to those of nonselective carious tissue removal and restoration, even when it was used to treat multisurface medium/large dentine le- sions. Treatment success was higher when daily toothbrushing was supervised.

For the treatment of deep caries lesions in primary teeth, techniques involving selective carious tissue removal showed a reduction in the incidence of pulp exposure in asymptomatic, vital, carious teeth. Still, the final, patient-relevant outcome is pain and therefore avoiding pulpal complications. Thus, the HT with no carious tissue removal before placement of a preformed metal crown and the standard procedure for preformed metal crowns with nonselective carious tissue removal and often pulpotomy result in similarly high success rates [Santamaria et al., 2020].

Atraumatic restorative treatment (ART) seems to increase the risk of restoration failure as compared to conventional restorative approaches at a low-quality level of evidence, mainly for multisurface cavities. In contrast, ART/HVGIC showed to be an adequate management option for treating single-surface carious lesions in primary teeth [Santamaria et al., 2020].

The presence of caries lesions in primary teeth/molars should immediately trigger intensified general efforts to decrease caries activity via measures listed under the subheading "Prevention of ECC," e.g., plaque removal, topical fluoride application (through daily toothbrushing and fluoride varnish applications) and a reduced sugar consumption.

\section{Recommendations Regarding the Treatment of} Cavitated Carious Dentine Lesions at the Site Level

NRCC achieves comparable results to those of conventional direct restorations. NRCC might only be used for compliant patients (strength of recommendation: weak; consensus: agreement 50\%, neutral $37 \%$, disagreement $12 \% *$; median: $7 ;^{*} \neq 100 \%$ due to rounding).

Dentine caries lesions in primary teeth can be treated successfully with silver (diammine) fluoride $(\geq 30 \%)$, when no irreversible pulpal involvement is present (strength of recommendation: moderate; consensus: agreement $80 \%$, neutral $16 \%$, disagreement $4 \%$; median: 9).

The HT without carious tissue removal or selective caries excavation and restoration seems advantageous in comparison to complete nonselective carious tissue removal in primary teeth without irreversible pulpal involvement (strength of recommendation: moderate; consensus: agreement $71 \%$, neutral $25 \%$, disagreement $4 \%$; median: 9).

For multisurface cavities, ART may increase the risk of restoration failure as compared to conventional restora- 
tions. In occlusal lesions of primary teeth it can achieve acceptable results (strength of recommendation: weak; consensus: agreement $83 \%$, neutral $12 \%$, disagreement $4 \% *$; median: $9 ; * \neq 100 \%$ due to rounding).

In proximal surfaces of primary molars without irreversible pulpal inflammation, the HT is significantly more successful than caries removal and fillings or NRCC treatment (strength of recommendation: moderate; consensus: agreement $71 \%$, neutral $21 \%$, disagreement $9 \%$; median: $8 ;{ }^{*} \neq 100 \%$ due to rounding).

In primary molars, the conventional preformed preformed metal crowns with tooth preparation are more successful than multisurface fillings (strength of recommendation: moderate; consensus: agreement $75 \%$, neutral $16 \%$, disagreement $8 \% *$; median: $8 ;{ }^{*} \neq 100 \%$ due to rounding).

\section{Occlusal Caries in Permanent Posterior Teeth}

Occlusal surfaces in molars are the first and most frequent surfaces in permanent teeth affected by caries, especially due to their long eruption period presenting with infraposition to the occlusal plane. Thus, the prevention and treatment of caries in the pits and fissures of occlusal surfaces are of primary concern in children and adolescents [Carvalho et al., 1989, 2016]. Besides standard preventive methods such as toothbrushing with fluoridated toothpaste, additional in-office fluoride varnish applications and preventive sealants are effective but costly [Ahovuo-Saloranta et al., 2016, 2017; Akinlotan et al., 2018] . Thus, they should be adjusted to the caries risk of the individual or the respective group/population [Schwendicke et al., 2018; Slayton et al., 2018].

Fluoride varnish applications and sealants can also be used for active, noncavitated occlusal lesions as noninvasive treatment [Schwendicke et al., 2015; Wright et al., 2016; Urquhart et al., 2019]. The last, but also very successful treatment option for cavitated lesions are occlusal, defect-orientated fillings, mostly with resin composite and possibly a subsequent sealant for the remaining fissures in case of persisting caries risk [Schwendicke et al., 2015]. Furthermore, sealing microcavitated caries lesions (ICDAS 3) in occlusal and smooth surface with resin or glass ionomer sealant materials has recently been shown as an effective alternative to arrest progression of caries in permanent first molars [Muñoz-Sandoval et al., 2019].

\section{Preventive Recommendations}

The first approach to prevent occlusal caries in permanent molars and premolars should be brushing with fluoridated toothpaste $(\geq 1,000 \mathrm{ppm})$ (strength of recommen- dation: strong; consensus: agreement $87 \%$, neutral $12 \%$, disagreement $0 \% *$; median: $9 ;^{*} \neq 100 \%$ due to rounding).

There should be a specific focus on reaching the occlusal surfaces in the erupting permanent premolars and molars when brushing whilst they are below the occlusal plane (strength of recommendation: weak; consensus: agreement $91 \%$, neutral $4 \%$, disagreement $4 \% *$; median: $9 ;^{*} \neq 100 \%$ due to rounding).

Additional preventive fluoride varnish applications or the use of fluoride gels is advisable for teeth, individuals, groups, or populations with a higher caries risk, activity, or prevalence (strength of recommendation: moderate; consensus: agreement $87 \%$, neutral $12 \%$, disagreement $0 \% *$; median: $9 ;{ }^{*} \neq 100 \%$ due to rounding).

\section{Microinvasive Treatment Recommendations}

Due to their high costs, preventive sealants are especially recommended for teeth, individuals, groups, or populations with a higher caries risk, activity or prevalence (strength of recommendation: moderate; consensus: agreement $95 \%$, neutral $0 \%$, disagreement $5 \%$; median: 9).

Preventive sealants should be placed with low viscosity resin composites. For teeth during eruption and for problems with moisture control, GIC can be used (strength of recommendation: weak; consensus: agreement $95 \%$, neutral $0 \%$, disagreement $4 \% *$; median: 10 ; $* \neq 100 \%$ due to rounding).

Noncavitated occlusal caries lesions should be sealed if these cannot be inactivated with noninvasive care (strength of recommendation: weak; consensus: agreement $83 \%$, neutral $8 \%$, disagreement $8 \% *$; median: 9 ; $^{*} \neq$ $100 \%$ due to rounding).

To arrest noncavitated caries lesions in occlusal surfaces, nonoperative caries treatment can be used (strength of recommendation: weak; consensus: agreement $88 \%$, neutral $8 \%$, disagreement $4 \%$; median: 9).

\section{Invasive Treatment Recommendations}

Occlusal cavitated lesions should be restored with a filling, preferably defect-orientated composite after carious tissue removal, possibly followed by sealing the remaining fissures according to the caries risk. The traditional preparation "with extension for prevention" involving the whole fissure system is not advised. In case of deep caries lesions, selective carious tissue removal and a well-sealed restoration should be applied (strength of recommendation: weak; consensus: agreement $91 \%$, neutral $4 \%$, disagreement $4 \% *$; median: $9 ;^{*} \neq 100 \%$ due to rounding). 


\section{Conclusions}

As the caries process manifests itself in subclinical demineralization, initial caries lesions, and finally cavitation, it can be treated with nonoperative, micro- and minimally invasive measures. It is almost impossible to draw a clear cut-off point between prevention and therapy when caries is defined as a continuous process of a net imbalance of de- and remineralization. Therefore, the term "caries management" describes a comprehensive approach to control or reduce caries activity.

This is especially true for the primary and erupting permanent dentition in children and adolescents where the lifetime pathway for oral health is laid. Besides wellresearched and established preventive approaches or treatment of permanent teeth, the success rates in the primary dentition, especially for ECC, are suboptimal. The presented statements compile the best available evidence for a consensus on clinical recommendations on to how to intervene in the caries process in children. They are specifically intended to support decision making in practice, and although all recommendations are consented their evidence base is sometimes limited due to a lack of adequate research. Hence, the statements also highlight research gaps that should be filled.

\section{Statement of Ethics}

This paper complies with internationally accepted standards for research practice and reporting. As consensus statement, it does not involve human subjects, specimen or animals.

\section{Disclosure Statement}

A.B. received support for a researcher-led SR of sugar-free gum by Mars Wrigley. P.B. received an honorarium for consultancy on product development regarding future caries treatments in medi- cally compromised adults from Mayser Pharma. L.B. received for support for research protocols regarding dental adhesives from 3M ESPE, Ivoclar Vivadent, Dentsply Sirona, VOCO, Heraeus Kulzer, Kuraray Noritake, Ultradent, and Pulpdent and material samples for this research from 3M ESPE, Ivoclar Vivadent, Dentsply Sirona, VOCO, Heraeus Kulzer, Kuraray Noritake, Ultradent, Sweden \& Martina, DMG, and Pulpdent. R.A.G. received dental materials from DMG and from 3M Oral Care to support some of the research cited in this paper. D.J.M. received support for conference lecturing and attendance by dental manufacturing companies DMG, GC Corporation, and SDI. N.J.M.O. received financial support for a practice-based research network from Kuraray Europe. F.S. received an honorarium for consultancy on product development regarding future caries treatments, study support on radiopaque tagging of caries lesions, restoring teeth with selective caries excavation, and for organizing the IADR symposia on caries treatment from DMG. C.H.S. received research and travel support from SDI regarding the use of silver diammine fluoride. A.F.Z. received an honorarium for consultancy on product development regarding future caries treatments from Colgate, GreenMark, Calcivis, research support from Colgate, NIDCR, Greenmark, Delta Dental, Calcivis, and nonmonetary support (e.g., equipment, facilities, research assistants, and paid travel to meetings) for an IADR symposia on caries detection by Calcivis. D.T.Z. received an honorarium for consultancy Colgate Palmolive, Procter \& Gamble, Greenmark Biomedical Inc., GlaxoSmithKline, and Johnson \& Johnson and research support from Novatis Pharmaceuticals, Johnson \& Johnson, Univerlever, Hello Products, and Church and Dwight.

\section{Funding Sources}

Travel funds for participation in the consensus meeting were provided by the scientific organizations ORCA, EFCD, and DGZ.

\section{Author Contributions}

C.H. Splieth prepared this paper based on the according systematic reviews and statements discussed and voted on by all of the authors. This paper was finalized with S. Paris and F. Schwendicke. All of the other authors corrected and approved this paper.

\section{References}

Adair PM, Pine CM, Burnside G, Nicoll AD, Gillett A, Anwar S, et al. Familial and cultural perceptions and beliefs of oral hygiene and dietary practices among ethnically and socio-economicall diverse groups. Community Dent Health. 2004 Mar;21(1 Suppl): $102-11$.

Ahovuo-Saloranta A, Forss H, Hiiri A, Nordblad A, Mäkelä M. Pit and fissure sealants versus fluoride varnishes for preventing dental decay in the permanent teeth of children and adolescents. Cochrane Database Syst Rev. 2016 Jan;(1):CD003067.
Ahovuo-Saloranta A, Forss H, Walsh T, Nordblad A, Mäkelä M, Worthington HV. Pit and fissure sealants for preventing dental decay in permanent teeth. Cochrane Database Syst Rev. 2017 Jul;7:CD001830.

Akinlotan M, Chen B, Fontanilla TM, Chen A, Fan VY. Economic evaluation of dental sealants: A systematic literature review. Community Dent Oral Epidemiol. 2018 Feb;46(1):38-46.

Campus G, Ceballos L, Doméjean S, Ekstrand K. How to intervene in the caries process: occlusal caries in permanent posterior teeth. Caries Res. Forthcoming 2020.
Carvalho JC, Dige I, Machiulskiene V, Qvist V, Bakhshandeh A, Fatturi-Parolo C, et al. Occlusal Caries: Biological Approach for Its Diagnosis and Management. Caries Res. 2016; 50(6):527-42.

Carvalho JC, Ekstrand KR, Thylstrup A. Dental plaque and caries on occlusal surfaces of first permanent molars in relation to stage of eruption. J Dent Res. 1989 May;68(5):773-9.

Casamassimo PS, Thikkurissy S, Edelstein BL, Maiorini E. Beyond the dmft: the human and economic cost of early childhood caries. J Am Dent Assoc. 2009 Jun;140(6):650-7. 
Collado V, Pichot H, Delfosse C, Eschevins C, Nicolas E, Hennequin M. Impact of early childhood caries and its treatment under general anesthesia on orofacial function and quality of life : A prospective comparative study. Med Oral Patol Oral Cir Bucal. 2017 May;22(3):e333-41.

Ganesh A, Muthu MS, Mohan A, Kirubakaran R. Prevalence of Early Childhood Caries in India - A Systematic Review. Indian J Pediatr. 2019 Mar;86(3):276-86.

Innes NP, Ricketts D, Chong LY, Keightley AJ, Lamont T, Santamaria RM. Preformed crowns for decayed primary molar teeth. Cochrane Database Syst Rev. 2015 Dec;(12): CD005512.

Machiulskiene V, Campus G, Carvalho JC, Dige I, Ekstrand KR, Jablonski-Momeni A, Maltz M, Manton DJ, Martignon S, Martinez-Mier EA, Pitts NB, Schulte A, Splieth CH, Tenuta L, Ferreira Zandona A, Nyvad B. Terminology of dental caries and dental caries management: consensus report of a workshop organized by ORCA and Cariology Research Group of IADR. Caries Res. 2020;54:7-14.

Muñoz-Sandoval C, Gambetta-Tessini K, Giacaman RA. Microcavitated (ICDAS 3) carious lesion arrest with resin or glass ionomer sealants in first permanent molars: a randomized controlled trial. J Dent. 2019 Sep;88:103163.

Nyvad B, Fejerskov O. The caries control concept. In: Fejerskov O, Nyvad B, Kidd E, editors. Dental Caries: The disease and its clinical management. 3rd ed. Oxford: Wiley Blackwell; 2015. pp. 235-43.

Pitts N, Baez R, Diaz-Guallory C, et al. Early Childhood Caries: IAPD Bangkok Declaration. Int J Paediatr Dent. 2019 May;29(3): 384-6.

Quist V, Laurberg L, Poulsen A, Teglers PT. Class II restorations in primary teeth: 7-year study on three resin-modified glass ionomer cements and a compomer. Eur J Oral Sci. 2004 Apr;112:188-96.
Santamaria RM, Schmoeckel J, Basner R, Schüler E, Splieth CH. Caries Trends in the Primary Dentition of 6- to 7-Year-old Schoolchildren in Germany from 1994 to 2016: Results from the German National Oral Health Surveys in Children. Caries Res. 2019;53:659-66.

Santamaría RM, Mohamed HA, Gulsun G, Said MHD, Felix Gome Gz, Ferreira Zandona AG. How to intervene in the caries process: dentine caries in primary teeth. Caries Res. Forthcoming 2020.

Schmoeckel J, Gorseta K, Splieth CH, Juric H. How to intervene in the caries process: early childhood caries - a systematic review. Caries Res. 2020 Jan;7:1-11.

Schwendicke F, Jäger AM, Paris S, Hsu LY, Tu YK. Treating pit-and-fissure caries: a systematic review and network meta-analysis. J Dent Res. 2015 Apr;94(4):522-33.

Schwendicke F, Splieth CH, Thomson WM, Reda S, Stolpe M, Foster Page L. Cost-effectiveness of caries-preventive fluoride varnish applications in clinic settings among patients of low, moderate and high risk. Community Dent Oral Epidemiol. 2018 Feb;46(1):8-16.

Slayton RL, Urquhart O, Araujo MWB, Fontana $\mathrm{M}$, Guzmán-Armstrong $\mathrm{S}$, Nascimento $\mathrm{MM}$, et al. Evidence-based clinical practice guideline on nonrestorative treatments for carious lesions: A report from the American Dental Association. J Am Dent Assoc. 2018;149:83749.

Splieth CH, Santamaria RM, Basner R, Schüler E, Schmoeckel J. 40-Year Longitudinal Caries Development in German Adolescents in the Light of New Caries Measures. Caries Res. 2019;53(6):609-16.

Team DAJ. Epidemiologische Begleituntersuchungen zur Gruppenprophylaxe 2016. Bonn: Deutsche Arbeitsgemeinschaft für Jugendzahnpflege; 2017
Thomson WM. Public Health aspects of paediatric dental treatment under general anaesthetic. Dent J (Basel). 2016 Jun;4(2):20.

Tinanoff N, Baez RJ, Diaz Guillory C, Donly KJ Feldens CA, McGrath C, et al. Early childhood caries epidemiology, aetiology, risk assessment, societal burden, management, education, and policy: global perspective. Int J Paediatr Dent. 2019 May;29(3):238-48.

Toumba KJ, Twetman S, Splieth C, Parnell C, van Loveren C, Lygidakis NA. Guidelines on the use of fluoride for caries prevention in children: an updated EAPD policy document. Eur Arch Paediatr Dent. 2019 Dec;20(6):507-16.

Urquhart O, Tampi MP, Pilcher L, Slayton RL, Araujo MW, Fontana M, et al. Nonrestorative Treatments for Caries: Systematic Review and Network Meta-analysis. J Dent Res. 2019 Jan; 98(1):14-26.

Walia T, Salami AA, Bashiri R, Hamoodi OM, Rashid F. A randomised controlled trial of three aesthetic full-coronal restorations in primary maxillary teeth. Eur J Paediatr Dent. 2014 Jun;15(2):113-8.

Walsh T, Worthington HV, Glenny AM, Marinho VC, Jeroncic A. Fluoride toothpastes of different concentrations for preventing dental caries. Cochrane Database Syst Rev. 2019 Mar;3:CD007868.

Wright JT, Tampi MP, Graham L, Estrich C, Crall JJ, Fontana M, et al. Sealants for preventing and arresting pit-and-fissure occlusal caries in primary and permanent molars. Pediatr Dent. 2016;38(4):282-308.

Wyne AH. Early childhood caries: nomenclature and case definition. Community Dent Oral Epidemiol. 1999 Oct;27(5):313-5.

Xiao J, Alkhers N, Kopycka-Kedzierawski DT, Billings RJ, Wu TT, Castillo DA, et al. Prenatal oral health care and early childhood caries prevention: a systematic review and metaanalysis. Caries Res. 2019;53(4):411-21. 